\title{
A unique case of reversion to normal size of a maternal premutation FMR1 allele in a normal boy
}

\author{
Elisabetta Tabolacci ${ }^{1}$, Maria Grazia Pomponi ${ }^{1}$, Roberta Pietrobono ${ }^{1}$, Pietro Chiurazzi ${ }^{1}$ \\ and Giovanni Neri*,1
}

${ }^{1}$ Facolta di Medicina e Chirurgia, Institute of Medical Genetics, Catholic University, Rome, Italy

Fragile $X$ syndrome (FXS) is caused mostly by expansion and subsequent methylation of the CGG repeat in the 5'UTR of the FMR1 gene, resulting in silencing of the gene, absence of FMRP and development of the FXS phenotype. The expansion also predisposes the CGG repeat and the flanking regions to further instability that may lead to mosaics between a full mutation and a premutation or, rarely, a normal or deleted allele. Here, we report on a 10-year-old boy with no FXS phenotype, who has a normal CGG tract, although he inherited the maternal expanded allele that causes FXS in his two brothers. Southern blotting demonstrated that the mother carries a premutation allele ( $\sim 190 \mathrm{CGG})$, whereas the propositus shows a normal $5.2 \mathrm{~kb}$ fragment after HindIII digestion and a smaller $2.2 \mathrm{~kb}$ fragment after double HindIII-Eagl digestion, without any apparent mosaicism in peripheral blood leukocytes. PCR and sequence analysis of the FMR1 5'UTR revealed an allele of 43 repeats, with two interspersed AGG triplets in position 10 and 25 and an exceptional CCG triplet in position 17. This latter creates an abnormal Eagl site compatible with the smaller $2.2 \mathrm{~kb}$ fragment observed with Southern blotting. Haplotype analysis proved that the rearranged allele originated from the maternal expanded allele. To the best of our knowledge, this is the first nonmosaic case of reduction in the CGG tract of the FMR1 gene, resulting in a normal allele.

European Journal of Human Genetics (2008) 16, 209-214; doi:10.1038/sj.ejhg.5201949; published online 31 October 2007

Keywords: FMR1 gene; intragenic deletion; triplet repeat contraction

\section{Introduction}

The fragile $\mathrm{X}$ syndrome (FXS) (OMIM no. 300624) is associated with the presence of a $>200$ repeat expansion of a CGG trinucleotide sequence in the $5^{\prime}$ noncoding region of the FMR1 gene. ${ }^{1-4}$ The subsequent methylation of cytosines in the promoter and in the expanded repeat itself $^{5,6}$ causes the loss of FMR1 gene expression. ${ }^{7,8}$ This mutational mechanism accounts for the vast majority of FXS cases. Other mutations in the FMR1 gene have been reported: a de novo $\mathrm{T} \rightarrow \mathrm{A}$ missense mutation that converts

*Correspondence: $\operatorname{Dr}$ G Neri, Facolta di Medicina e Chirurgia, Institute of Medical Genetics, Catholic University, Largo F. Vito, 1, Rome 00168, Italy. Tel: + 39063054 449; Fax: + 39063050 031;

E-mail: gneri@rm.unicatt.it

Received 21 July 2007; revised 28 September 2007; accepted 2 October 2007; published online 31 October 2007 an isoleucine at codon 367 to asparagine, identified in an individual with some of the clinical features of the FXS; ${ }^{9}$ an inherited 2-base pair substitution that alters the splice acceptor site of exon $2^{10}$ and several partial or complete deletions, ranging in size from 1 to $13 \mathrm{Mb} .^{10-19}$

The polymorphic CGG sequence expansion apparently predisposes the repeat itself, as well as its flanking regions, to further instability that may lead to mosaic conditions. Mosaicism for a full mutation and a premutation is not uncommon in FXS, occurring with a frequency variably estimated at $12-41 \%$ of all cases. ${ }^{20-22}$ On the other hand, mosaicism for a full mutation with a partially deleted or with a normal allele has been rarely reported in cases of FXS. ${ }^{18}$ Direct sequencing of the deletion breakpoint and Southern blotting analysis suggest that two independent events occur in the regression from a full mutation to a normal or a deleted allele: the deletion encompassing the 
CGG tract and the loss of DNA methylation. ${ }^{23}$ The transcription of an unmethylated and reduced FMR1 allele was not impaired in lymphocytes of a mosaic FXS patient. $^{24}$

The molecular mechanisms responsible for the CGG repeat expansion and, more rarely, for reductions in the FMR1 gene, are complex and still unclear. One of these is probably slipped strand mispairing (SSM): DNA polymerase replication of GC-rich repetitive sequences is error-prone because of transient detachment of the newly synthesized strand and mispairing during DNA replication, due to the repetitive nature of the CGG stretch. ${ }^{25,26}$

Here, we describe a normal boy belonging to an FXS family, in whom a non-mosaic, apparently normal FMR1 allele resulted from reduction of the expanded maternal allele. The patient has two FXS brothers with a methylated full mutation. Their clinically normal mother has a large premutation of $\sim 190$ CGG repeats. To the best of our knowledge, this case represents the first documented instance of a reversion to normal size of an expanded FMR1 allele, not in a mosaic state, possibly arising in the maternal germ line.

\section{Materials and methods}

\section{Southern blotting and CGG PCR analysis}

Informed consent was obtained for all subjects participating in this study. Blood was drawn from various family members, as shown on the pedigree (Figure 1). Genomic DNA was isolated from $10 \mathrm{ml}$ of peripheral blood leukocytes using standard procedures and $10 \mu \mathrm{g}$ of DNA was digested with HindIII and/or EagI (New England Biolabs). Digested DNA and a size marker were run on a $0.8 \%$ agarose gel with $1 \times$ TAE buffer, blotted on Amersham Hybond $\mathrm{N}+$ nylon membrane and hybridized with radioactive Ox1.9 probe. $^{27}$ After overnight hybridization and subsequent washing, radioactive filters were exposed to films at $-80^{\circ} \mathrm{C}$ with reinforcing screens before development.

PCR amplification of the CGG repeats was carried out for all family members with fluorescent primers (see Figure 1). The amplification products were separated on capillary gel electrophoresis using an ABI 3130 sequencer and analyzed with GenMapper v4.0.

\section{Sequence analysis}

The DNA of the proband and of a normal control male was amplified, using the following primers for the FMR1 gene promoter, that span the CGG repeat region (GenBank L29074): 1F, 5'-AACTGGGATAACCGGATGCAT-3' and 2R, 5'-GGCAAAGCCAAAGTGAAGGC-3'; 3F, 5'-CCGTTTCGGT TTCACTTCCG-3' and 4R, 5'-GGTTACCGCGAAAGATGT TC-3'. PCR amplifications were performed as follows: 30 cycles $\left(1 \mathrm{~min}\right.$ at $95^{\circ} \mathrm{C} ; 1 \mathrm{~min}$ at $55^{\circ}-60^{\circ} \mathrm{C}$, depending on the couple of primers; $1 \mathrm{~min}$ at $72^{\circ} \mathrm{C}$ ) with $10 \%$ of DMSO,

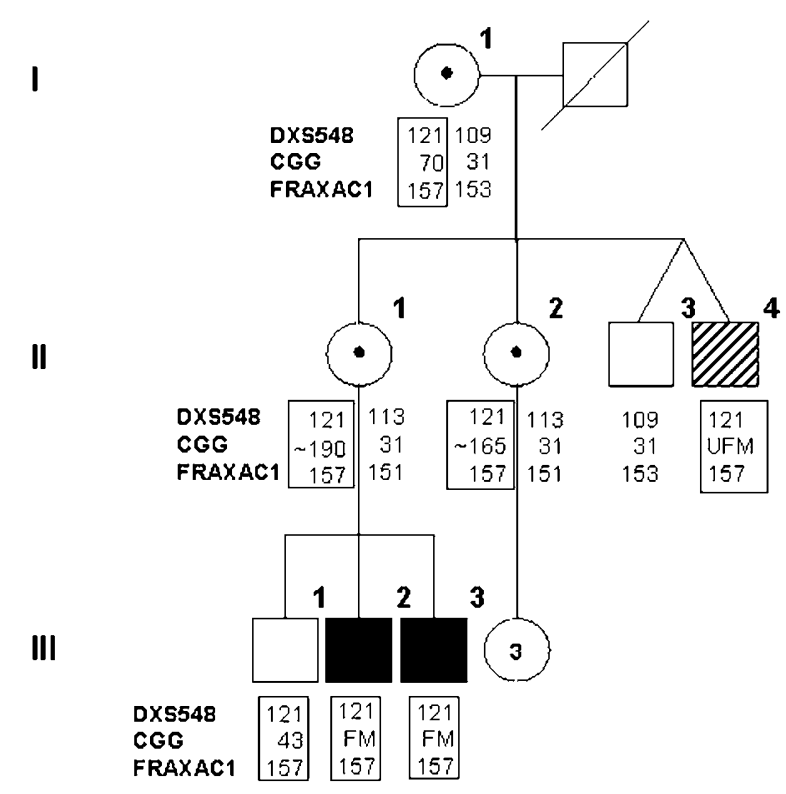

Figure 1 Pedigree of the patient's family with the proband (III-1) indicated by an arrow. Black squares indicate affected males (III-2, III-3) with a methylated full mutation (FM), while II-4 had normal intelligence and carries an unmethylated full mutation (UFM). Haplotype analysis of the family with polymorphic markers DXS548 and FRAXAC1 is indicated below each individual's symbol and shows that the proband has the same haplotype $(121-157)$ of his two affected brothers (III-2 and III-3) and of the maternal uncle (II-4). The number of CGG repeats is also indicated between marker DXS548, which maps $150 \mathrm{~kb}$ upstream of the CGG repeat, and marker FRAXAC1, which maps downstream in intron 1.

$200 \mu \mathrm{M}$ of dNTPs, $2.0 \mathrm{mM}$ of $\mathrm{MgCl}_{2}, 1 \mathrm{U}$ of Taq polymerase and $1 \mathrm{pmol}$ of each primer in a final volume of $30 \mu \mathrm{l}$. A total of $10 \mu \mathrm{l}$ of each PCR product was visualized with ethidium bromide on a $1.2 \%$ agarose gel. To check for the presence of the extra EagI site, part of the amplification product was digested with this enzyme and the digested PCR product was separated on $2 \%$ agarose gel.

The $3 \mathrm{~F}-4 \mathrm{R}$ amplicon, that in the proband DNA contains the CGG repeats and the second $\mathrm{EagI}$ site, was sequenced along with that of a normal control male. Both forward and reverse strands were sequenced using the BigDye Terminator Cycler Sequence kit and protocol (Applied Biosystems). The reactions were denatured for $6 \mathrm{~min}$ at $96^{\circ} \mathrm{C}$, followed by 27 cycles of $10 \mathrm{~s}$ at $96^{\circ} \mathrm{C}, 5 \mathrm{~s}$ at $50^{\circ} \mathrm{C}$, $4 \mathrm{~min}$ at $60^{\circ} \mathrm{C}$ and extension of $10 \mathrm{~s}$ at $60^{\circ} \mathrm{C}$. Samples were then purified with $3 \mathrm{M}$ sodium acetate $(\mathrm{pH}$ 5.0) and ethanol, denatured and loaded into the ABI 3130 DNA sequencer.

\section{Haplotype analysis}

A segregation study was performed on all family members identified in Figure 1. PCR amplification of the polymorphic markers DXS548 and FRAXAC1 was carried out with fluorescent primers. ${ }^{4,28}$ Amplifications were 
performed as follows: 30 cycles $\left(1 \mathrm{~min}\right.$ at $95^{\circ} \mathrm{C}$; $1 \mathrm{~min}$ at $55^{\circ} ; 1 \mathrm{~min}$ at $72^{\circ} \mathrm{C}$ ) with $200 \mu \mathrm{M}$ of dNTPs, $2.0 \mathrm{~mm}$ of $\mathrm{MgCl}_{2}$, $1 \mathrm{U}$ of Taq polymerase and 1 pmol of each primer in a final volume of $20 \mu \mathrm{l}$. A total of $10 \mu \mathrm{l}$ of each PCR product was visualized with ethidium bromide on a $1.2 \%$ agarose gel. Alleles were resolved on capillary gel electrophoresis using an ABI 3130 sequencer and analyzed with GenMapper v4.0.

\section{RNA extraction and quantitative RT-PCR}

Total RNA was extracted from proband and control male lymphoblastoid cell lines with the single-step acid phenol method, using Trizol (Invitrogen). Two micrograms of total RNA were retro-transcribed to cDNA by MoMLV-RT (Invitrogen) and then used for quantitative fluorescent RT-PCR. ${ }^{29}$

\section{Results}

Figure 1 shows the pedigree of the family with the corresponding haplotype and CGG analysis. The proband (III-1) was a 10-year-old boy referred for genetic testing, because FXS had been previously diagnosed in his two younger brothers (III-2 and III-3). His physical and psychomotor development was normal.

Southern blotting analysis of genomic DNA at the FMR1 locus, together with CGG repeat segregation study, was extended to relevant members of the family. The maternal grandmother (I-1) was found to be carrier of a 31 CGG normal allele and a premutation allele of approximately 70 CGGs. Her premutation was associated with premature ovarian failure (POF) with menopause at 40 years, and was transmitted in four different manners to the offspring. Subject II-4 was apparently not mentally retarded and carried an unmethylated full mutation (UFM) with an amplification of $0.7-1.0 \mathrm{~kb}$ (corresponding to approximately 265-365 CGGs). The molecular and epigenetic characterization of this UFM will be reported elsewhere. His twin brother (II-3) inherited the normal maternal allele of 31 CGGs. The proband's maternal aunt (II-2) had a 31 CGG paternal allele and a $0.4 \mathrm{~kb}$ premutation allele $(\sim 165$ CGGs), and the proband's unaffected mother (II-1) carried a 31 CGG paternal allele and a $0.5 \mathrm{~kb}$ band, corresponding to a large premutation of approximately 190 CGGs (Figure 2, lane 3). This premutation allele was further expanded in her FXS sons (III-2 and III-3), who carried a $0.7-2.4 \mathrm{~kb}$ (265-830 CGGs) and $1.2-2.3 \mathrm{~kb}(430-795$ CGGs) methylated full mutations, respectively. Upon parental request, the proband was also tested to rule out the possibility of being a premutation carrier. Surprisingly, on the Southern blotting after HindIII/EagI double digestion, we observed a smaller band $(2.2 \mathrm{~kb})$ compared with the expected $2.5 \mathrm{~kb}$ band (Figure 2, lane 4). We first suspected a deletion that was ruled out by Southern blotting analysis with HindIII digestion alone, showing a

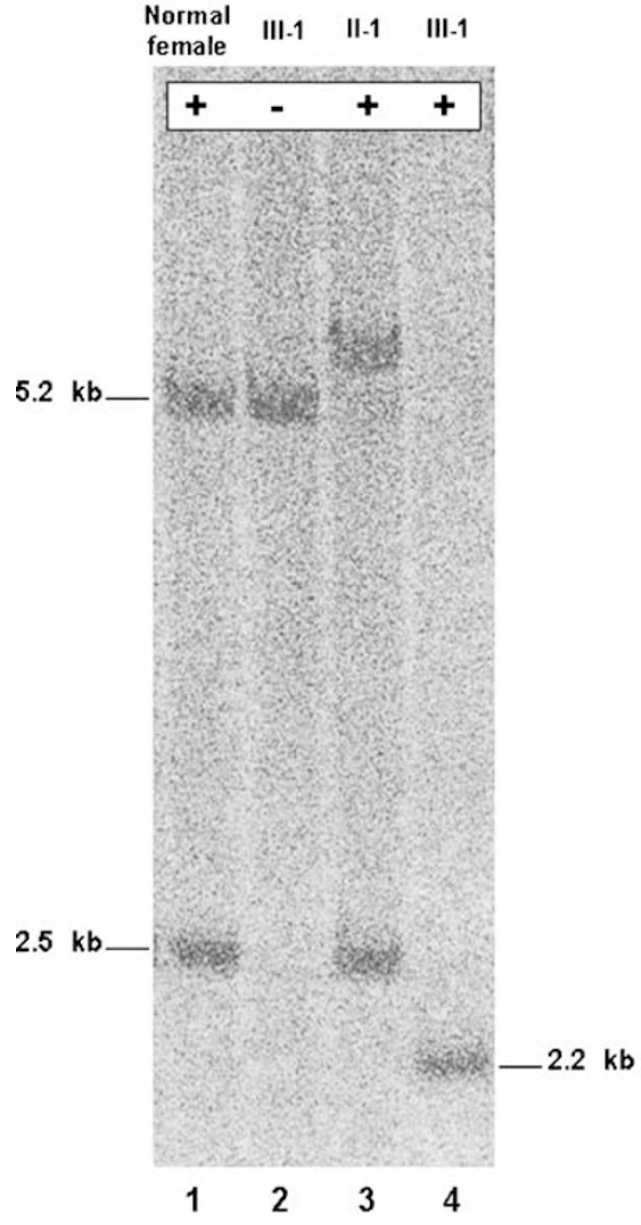

Figure 2 Southern blot analysis of the proband's and his mother's DNAs digested with Hindlll and Eagl $(+)$ or Hindlll alone $(-)$, hybridized with radioactive Ox1.9 probe. Lane 1 corresponds to a normal control female with two normal bands of 2.5 and $5.2 \mathrm{~kb}$ bands corresponding to the active and inactive $\mathrm{X}$ chromosomes, respectively. Lane 3 corresponds to the proband's mother (II-1), carrier of a large premutated allele of $0.5 \mathrm{~kb}(\sim 190 \mathrm{CGGs})$. Lanes 2 and 4 correspond to proband III-1, cut with HindIII alone $(5.2 \mathrm{~kb})$ or HindlII and Eagl, respectively. Only double digestion (lane 4 ) reveals the presence of a $2.2 \mathrm{~kb}$ fragment, $300 \mathrm{bp}$ shorter than expected.

normal band of $5.2 \mathrm{~kb}$ (Figure 2, lane 2). The absence of extra bands, confirmed on repeated blots with longer exposures, ruled out the possibility of mosaicism with larger alleles in the range of premutation and/or full mutations, either methylated or unmethylated.

PCR amplification of the CGG repeat showed the presence of 43 triplets, which did not correspond to the normal maternal allele. These findings suggested that the expanded maternal allele had undergone a partial deletion of the CGG repeat, with the incidental introduction of an extra EagI restriction site (CGGCCG) downstream the canonical site, which maps in the CpG island of the FMR1 promoter. Our hypothesis was confirmed by sequence analysis of the CGG repeat that showed a sequence of 43 


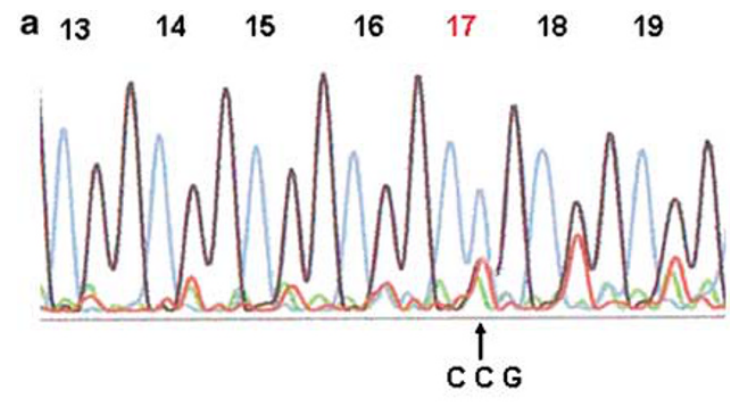

b

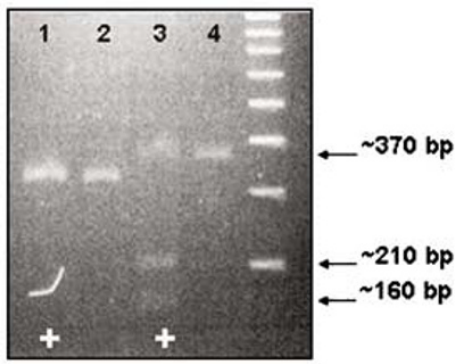

Figure 3 Analysis of CGG repeats sequence with primers $3 \mathrm{~F}$ and $4 R$. (a) Sequence analysis of the index case shows a 43 triplet repeat allele with two interspersed AGG triplets on position 10 and 25 (data not shown) and a CCG triplet on position 17. This unusual variant introduces a second Eagl restriction site (CGGCCG). (b) The presence of an extra Eagl site is confirmed by cutting the PCR product with Eagl $(+)$. The uncut PCR products of a control male $(\sim 330 \mathrm{bp})$ and of the index case $(\sim 370 \mathrm{bp})$ can be seen in lanes 2 and 4 , respectively, but while the fragment of the control male is not cut by Eagl (lane 1), two fragments of 210 and $160 \mathrm{bp}$ are visibile when the proband's DNA is digested with Eagl (lane 3). In lane 3, a residual uncut band of $370 \mathrm{bp}$ reflects a partial digestion.

triplets with an exceptional CCG repeat at position 17, corresponding to the postulated EagI extra site (Figure 3a). Furthermore, two AGG repeats were observed at positions 10 and 25, respectively. The presence of the extra EagI site in the proband's DNA accounted for the smaller $(2.2 \mathrm{~kb})$ HindIII/EagI fragment observed on the Southern blotting hybridized with the Ox1.9 probe. This result was confirmed by digesting with EagI the PCR product spanning the CGG repeat of the proband and of a normal male (Figure $3 b$ ). Sequence analysis of the FMR 1 promoter region demonstrated the presence of the canonical EagI restriction site. This finding was also confirmed by digesting the PCR product of the FMR1 promoter with EagI, showing the expected normal band both in the proband and in a normal control male (data not shown).

Haplotype analysis of polymorphic markers DXS548 and FRAXAC1, flanking the CGG repeat, demonstrated that the rearranged allele of the proband was derived from the maternal expanded allele, the same that had undergone expansion in his two FXS brothers (Figure 1). Thus, our case represents a bona fide example of reverse mutation from an expanded FMR1 allele (in the mother) to a normalsize allele (in the offspring). Results of haplotype analysis exclude the occurrence of unequal crossing-over and suggest the occurrence of a contraction event in cis in the maternal germline. Finally, measurement of FMR1 mRNA by real-time RT-PCR demonstrated a normal level in the proband, relative to a normal control male, in accordance with his normal phenotype (data not shown).

\section{Discussion}

We report on a normal boy belonging to an FXS family, probably representing the first described case of reversion to normal of an expanded CGG repeat in the FMR1 gene, with no evidence of mosaicism. This reduction resulted in a 43-repeat allele, containing an exceptional CCG triplet at position 17, which introduced an extra EagI restriction site. This unique situation would have gone unnoticed with PCR analysis or with HindIII digestion only, and was clarified with double HindIII/EagI digestion, followed by Southern blotting analysis. An unequal crossing-over event was excluded through the analysis of polymorphic markers, DXS548 (about $150 \mathrm{~kb}$ upstream of the CGG repeat) and FRAXAC1 (in intron 1). The proband and his two affected brothers, all inherited the same haplotype (121157) present in the maternal expanded allele. We think that the most likely explanation of this apparent inconsistency is a sporadic mutation in the maternal germline. However, we cannot rule out the possibility that the reduced allele was already present in a low-level mosaic state in the mother or that the reduction took place during early embryogenesis.

In this family, there is another peculiarity, namely the presence of an apparently normal male (II-4) harboring an UFM, which will be the object of a separate report. This latter finding might be due to mosaicism in the germline of I-1, who may be a carrier of larger premutations, transmitted to her daughters (II-1 and II-2), as well as UFM such as that inherited by II-4. The contemporary presence of a reduced allele in the proband III- 1 and of an UFM in his uncle II-4 is exceptional, although it is difficult to envisage a common cause underlying the two events.

FXS is most often associated with the presence of an expanded CGG repeat sequence within the first exon of the FMR1 gene, which usually results in local DNA hypermethylation and transcrptional silencing of the gene. While this mutational mechanism is largely prevalent, others have been described, including point mutations and deletions of variable size. Deletions, like expansions, could be the consequence of the instability of the FMR1 CGG repeats, once they exceed a certain size, around 55-60 CGG triplet repeats. Some patients have been reported with a mosaicism for a full mutation in association with an allele of normal size or an allele of reduced size, indicating that the instability is not restricted to the repeat itself but may extend to the flanking regions. In our case, there is no 
apparent mosaicism in lymphocytes and the reduction event may have originated in the maternal germline. The phenotypically normal mother appears to carry a large premutation in peripheral blood leukocytes, with no evidence of a reduced allele. On the other hand, from the analysis of intact ovaries of full mutation fetuses, Malter et $a l^{30}$ demonstrated that only UFM alleles can be detected in oocytes. Similarly, the testis of a 13-week full mutation fetus showed no evidence of premutations, whereas a 17 week full mutation fetus exhibited some germ cells with premutations. ${ }^{30}$ This study suggested that expanded alleles may contract in the immature testes. Thus, as already mentioned above, we cannot exclude the possibility that the proband inherited an expanded allele from his mother, which underwent a reversion to normal size during the early stages of embryogenesis.

The presence of deletions encompassing the CGG repeat, as well as sequences flanking the CGG tract, has been noted in a small number of FXS males. ${ }^{12,15,16,31}$ Interestingly, in many of these events, the $5^{\prime}$ breakpoint mapped to a 'deletion hotspot' region. ${ }^{31}$ It was demonstrated that CpG methylation and DNA replication may actually mediate the formation of these deletion events. ${ }^{32}$ Also, stabilizing AGG interruptions can be lost when replicationmediated CGG deletions occur. On characterizing a small deletion, it was noted that the location of the $5^{\prime}$ breakpoints in their patients corresponded to a very CpG-rich area. ${ }^{31}$ Therefore, we surmised that a CpG-rich region may be predisposed to rearrangements, resulting in deletions which encompass the promoter sequence preceding the CGG repeat tract. This also suggests that such deletions may occur as a result of intramolecular recombination involving direct repeats or double-strand break repair between the promoter region and the CGG repeat tract. On the other hand, SSM results in an increase or decrease of CGG repeat number depending on misalignment of either the nascent or template strand. The mutation rate is inversely correlated to the size of the repeat unit ${ }^{33}$ and directly related with the total repeat length. ${ }^{34}$ An 'expansion bias' would be expected, as amplifications increase the frequency of further slippage. However, reductions are also to be expected and, in fact, one such instance was observed in a normal FMR1 allele of 54 CGG repeats. ${ }^{35}$ An alternative mechanism could be represented by intramolecular recombination among prematurely terminated sequences and Okazaki fragments, resulting from the difficulty of the DNA polymerase to replicate repetitive GC-rich sequences. ${ }^{36}$ Unequal crossing-over between sister chromatids should also be considered, but this hypothesis has been ruled out in our case.

A relevant question posed by our findings relates to the origin of the CGG deletion, as the mother does not have a deleted allele in her somatic cells. This suggests that the CGG contraction was de novo, likely resulting from an intramolecular event involving the expanded repetitive tract either in the maternal germline or during the early embryogenesis of III-1. This deletion did not include the flanking regions of the CGG repeat and left intact the coding region of the gene, thus allowing normal transcription and production of the FMRP protein. This explains the normal phenotype of the proband. If we had performed only the HindIII digestion, yielding an apparently normal $5.2 \mathrm{~kb}$ band on Southern blotting, we would have considered the proband a normal male who inherited the normal FMR1 allele of his mother. However, the methylation status was tested by HindIII/EagI double digestion and revealed a $2.2-\mathrm{kb}$ band, about $300 \mathrm{bp}$ shorter than the expected $2.5 \mathrm{~kb}$ band. By PCR, the number of triplet repeats was found to be 43 , that is, different from 31, present in the normal maternal allele. By chance, the reduction event introduced an extra EagI restriction site within the CGG tract, as demonstrated by sequence analysis and EagI digestion of the PCR products of the FMR1 promoter and 5'UTR region. Deletion events across the FMR1 locus appear to be infrequent, but they will be occasionally found during routine molecular investigation of patients with developmental delay of unknown causes. The only reliable test that would detect such cases is a Southern blotting assay with double HindIII/EagI digestion. When counseling carriers of CGG tract expansions who have apparently normal offspring, PCR fragment length and haplotype analysis, as well as double HindIII/EagI digestion, may be considered to avoid missing a contraction event, such as the one reported here.

\section{Acknowledgements}

This work was supported by TELETHON grant (GGP06224), PRIN 2005 grant (no. 2005060575) and Conquer Fragile $X$ Foundation grant to GN.

\section{References}

1 Kremer EJ, Pritchard M, Lynch $\mathrm{M}$ et al: Mapping of DNA instability at the Fragile $\mathrm{X}$ to a trinucleotide repeat sequence p(CGG)n. Science 1991; 252: 1711-1714

2 Oberlé I, Rousseau F, Heitz D et al: Instability of a 550-base pair DNA segment and abnormal methylation in fragile $\mathrm{X}$ syndrome. Science 1991; 252: 1097-1102.

$3 \mathrm{Yu}$ S, Pritchard M, Kremer E et al: Fragile X genotype characterized by an unstable region of DNA. Science 1991; 252: 1179-1181.

4 Verkerk AJMH, Pieretti M, Sutcliffe JS et al: Identification of a gene (FMR-1) containing a CGG repeat coincident with a breakpoint cluster region exhibiting length variation in fragile $\mathrm{X}$ syndrome. Cell 1991; 65: 905-914.

5 Vincent A, Heitz D, Petit C, Kretz C, Oberlé I, Mandel JL: Abnormal pattern detected in fragile $\mathrm{X}$ patients by pulsed field gel electrophoresis. Nature 1991; 329: 624-626.

6 Hansen RS, Gartler SM, Scott CR, Chen SH, Laird CD: Methylation analysis of CGG sites in the CpG island of the FMR1 gene. Hum Mol Genet 1992; 1: 571-578.

7 Pieretti M, Zhang F, Fu YH et al: Absence of expression of the FMR1 gene in fragile X syndrome. Cell 1991; 66: 817-822. 
8 Sutcliffe JS, Nelson DL, Zhang F et al: DNA methylation represses FMR-1 transcription in fragile X syndrome. Hum Mol Genet 1992; 1: $397-400$

9 de Boulle K, Verkerk AJMH, Reyniers E et al: A point mutation in the FMR1 gene associated with fragile $\mathrm{X}$ mental retardation. Nat Genet 1993; 3: 31-35.

10 Lugenbeel KA, Peier AM, Carson NL, Chudley AE, Nelson DL: Intragenic loss of function mutations demonstrate the primary role of FMR1 in fragile X syndrome. Nat Genet 1995; 10: 483-485.

11 Gronskov K, Hjalgrim $\mathrm{H}$, Bjerager MO, Brondum-Nielsen K: Deletion of all CGG repeats plus flanking sequences inFMR1 does not abolish gene expression. Am J Hum Genet 1997; 61: 961 -967.

12 Hammond LS, Macias MM, Tarleton JC, Shashidahar Pai G: Fragile X syndrome and deletion in FMR1: new case and review of the literature. Am J Med Genet 1997; 72: 430-434.

13 Wolff DJ, Gustashaw KM, Zurcher V et al: Deletion in Xq26.3q27.3 including FMR1 result in a severe phenotype in a male and variable phenotypes in females depending upon the $\mathrm{X}$ inactivation pattern. Hum Genet 1997; 100: 256-261.

14 Orrico A, Galli L, Dotti MT, Plewnia K, Censini S, Federico A: Mosaicisism for full mutation and normal-sized allele of the FMR1 gene: a new case. Am J Med Genet 1998; 78: 341-344.

15 Grasso M, Faravelli F, Nigro CL et al: Mosaicis for the full mutation and a microdeletion involving the CGG repeat and flanking sequences in the FMR1 gene in eight fragile X patients. Am J Med Genet 1999; 85: 311-316.

16 Petek E, Kroisel PM, Schuster M, Zierler H, Wagner K: Mosaicism in a fragile X male including a de novo deletion in the FMR1 gene. Am J Med Genet 1999; 84: 229-232.

17 Garcia Arocena D, de Diego Y, Oostra BA, Willemsen R, Mirta Rodriguez M: A fragile $\mathrm{X}$ case with an amplification/deletion mosaic pattern. Hum Genet 2000; 106: 366-369.

18 Fan H, Booker JK, McCandless SE, Shashi V, Fleming A: Mosaicism for an FMR1 gene deletion in a fragile X female. Am J Med Genet 2005; 136: 214-217.

19 Han XD, Powell BR, Phalin JL, Chehab FF: Mosaicism for a full mutation, permutation and deletion of the CGG repeats results in $22 \%$ FMRP and elevated FMR1 mRNA levels in a high-functioning fragile X male. Am J Med Genet 2006; 140: 1463-1471.

20 Nolin SL, Glicksman A, Houck Jr GE, Brown WT, Dobkin CS: Mosaicism in fragile X affected males. Am J Med Genet 1994; 51: 509-512.

21 Rousseau F, Heitz D, Tarleton J et al: A multicenter study on genotype-phenotype correlations in the fragile $\mathrm{X}$ syndrome, using direct diagnosis with probe StB12.3: the first 2253 cases. Am J Hum Genet 1994; 55: 225-237.
22 Merenstein SA, Sobesky WE, Taylor AK, Riddle JE, Tran HX, Hagerman RJ: Molecular-clinical correlations in males with an expanded FMR1 mutation. Am J Med Genet 1996; 64: 388-394.

23 Weiss A, Keshet I, Razin A, Cedar H: DNA demethylation in vitro: involvement of RNA. Cell 1996; 86: 709-718.

24 de Graaff E, De Vries BBA, Willemsen $\mathrm{R}$ et al: The fragile $\mathrm{X}$ phenotype in a mosaic male with a deletion showing expression of the FMR1 protein in 28\% of the cells. Am J Med Genet 1996; 64: $302-308$.

25 Levinson G, Gutman GA: Slipped strand mispairing: a major mechanism for DNA sequence evolution. Mol Biol Evol 1987; 4: 203-221.

26 Chiurazzi P, Kozak L, Neri G: Unstable triplets and their mutational mechanism: size reduction of the CGG repeat vs germline mosaicism in the fragile X syndrome. Am J Med Genet 1994; 51: 517-521.

27 Nakahori Y, Knight SJL, Holland J et al: Molecular heterogeneity of the fragile X syndrome. Nucleic Acids Res 1991; 19: $4355-4359$.

28 Richards RI, Holman K, Kozman H et al: Fragile X syndrome: genetic localization by linkage mapping of two microsatellite repeats FRAXAC1 and FRAXAC2 which immediately flank the fragile site. J Med Genet 1991; 28: 818-823.

29 Pietrobono R, Pomponi MG, Tabolacci E, Oostra BA, Chiurazzi P, Neri G: Quantitative analysis of DNA demethylation and transcriptional reactivation of the FMR1 gene in fragile X cells treated with 5-azadeoxycytidine. Nucleic Acids Res 2002; 30: $3278-3285$

30 Malter HE, Iber JC, Willemsen R et al: Characterization of the full fragile X syndrome mutation in fetal gametes. Nat Genet 1997; 15: $165-169$.

31 de Graaff E, Rouillard P, Willems PJ, Smits AP, Rousseau F, Oostra BA: Hotspot for deletions in the CGG repeat region of FMR1 in fragile X patients. Hum Mol Genet 1995; 4: 45-49.

32 Edamura KN, Pearson CE: DNA methylation and replication: implications for the 'deletion hotspot' region of FMR1. Hum Genet 2005; 118: 301-304.

33 Schloetterer C, Tautz D: Slippage synthesis of imple sequence DNA. Nucleic Acids Res 1992; 20: 211-215.

34 Weber JL, Wong C: Mutation of human short tandem repeats. Hum Mol Genet 1993; 2: 1123-1128.

$35 \mathrm{Fu} \mathrm{YH,} \mathrm{Kuhl} \mathrm{DPA,} \mathrm{Pizzuti} \mathrm{A} \mathrm{et} \mathrm{al:} \mathrm{Variation} \mathrm{of} \mathrm{the} \mathrm{CGG} \mathrm{repeat} \mathrm{at}$ the fragile $\mathrm{X}$ site results in genetic instability: resolution of the Sherman paradox. Cell 1991; 67: 1047-1058.

36 Kuhl DPA, Caskey CT: Trinucleotide repeats and genome variation. Curr Opin Genet Dev 1993; 3: 404-407. 